\title{
Trametinib Monotherapy for the Treatment of Brain Metastasis from NSCLC: A Case Report and Literature Review
}

\section{Tianfan Pan}

Jiangyin People's Hospital

Xiangzhong Huang ( $\sim$ hxzdoc652@163.com )

Jiangyin People's Hospital https://orcid.org/0000-0003-4141-6160

\section{Research Article}

Keywords: Trametinib, NSCLC, Brain metastasis, Genetic mutation

Posted Date: May 10th, 2021

DOl: https://doi.org/10.21203/rs.3.rs-484320/v1

License: (c) (i) This work is licensed under a Creative Commons Attribution 4.0 International License.

Read Full License 


\section{Abstract}

\section{Background:}

BRAF mutation mostly was observed in lung adenocarcinoma, accounting for about $4.9 \%$. It mainly including V600E mutation and other rare mutations, among which non-V600E positive accounted for about $43.3 \%$.

\section{Case presentation:}

We report a case that postoperative recurrence NSCLC with brain metastasis and multiple lymph nodes metastasis, who had been developed hemiplegia. The gene mutation was positive for BRAF non-V600E and a partial response was observed despite brain and multiple lymph nodes lesions after trimetinib monotherapy.

\section{Conclusion:}

Our case shows that trimetinib could potentially be a promising therapeutic agent for chemotherapyrefractory BRAF non-V600E mutant NSCLC patients.

\section{Background}

The primary treatment for advanced non-small-cell lung cancer (NSCLC) has been updated from traditional chemotherapy and radiotherapy to targeted therapy and immunotherapy based on the pathological subtypes and gene sequencing results. We report a case of BRAF non-V600E mutation advanced NSCLC with retroperitoneal, hilar lymph nodes, and brain metastases in partial response after a short course of trametinib monotherapy.

\section{Case Presentation}

A 71-year-old never-smoker man underwent left upper lobectomy and thymectomy in July 2018. Pathology reported a (left lung) adenocarcinoma. The tumor invaded the pleura and thymic lymph nodes; thus 6 cycles of pemetrexed $800 \mathrm{mg}$ day 1 (d1) and carboplatin $400 \mathrm{mg}$ (d1) were subsequently delivered until January 2019. Then he accepted the accurate Gamma Knife (GK) radiosurgery to the lesions in the upper left side of the mediastinum (DT 38.4 GY/12F) in February 2019. Then the patient took Chinese medicine to recuperate, the specific details were unknown.

A computed tomography (CT) scan performed 18 months after surgery (January 2020) detected multiple lung and lymph nodes lesions (Fig. 1囚-囚), and CEA level increased to $14.8 \mathrm{ng} / \mathrm{ml}$, and CA153 level increased to $28.6 \mu / \mathrm{ml}$ and CA125 level increased to $47.8 \mu / \mathrm{ml}$. Ultrasound-guided needle biopsy of the intraperitoneal lymph node biopsy was performed on January 23. Pathology reported metastatic adenocarcinoma originated from lung. Immunohistochemically, the tumor cells tested positive for cytokeratin 7 (CK7), NapsinA, Ki-67 (labeling index =30\%), P40, and CKAE1/AE3 and the tumor cells were 
negative for cytokeratin 20 (CK20) and CK5/6. The next generation sequencing (NGS) was performed and the molecular results from intraperitoneal lymph node tissue biopsy did not show any abnormality (EGFR, KRAS, NRAS, ALK, ROS1, MET, HER-2, FGFR2, NTRK1, NTRK2, RET, PIK3CA) except BRAF exons 15 (p.D594N pathogenicity mutation). The patient developed symptoms of right progressive hypotonia to grade 1 and distortion of right commissure. Magnetic resonance imaging (MRI) of head showed that there was a mass (about $1.3 \mathrm{~cm}$ in diameter) in the left frontal lobe with brain edema, which was deemed metastatic (Fig. 1区). Furosemide and $20 \%$ mannitol were used to treat cerebral edema and the muscle tension recovered to grade 2 . The patient refused the treatment of whole brain radiotherapy and systemic chemotherapy.

The patient started to take trimetinib ( $2 \mathrm{mg}$ qd po) based on the molecular results from February 2, 2020. There have been systemic pruritus and hand-foot syndrome during the treatment, which was evaluated as grade 2 adverse reaction according to Common Terminology Criteria for Adverse Events (CTCAE) 5.0. and no other adverse reactions were observed. He got relief after taking positive measures. A CT scan performed 8 months after medication (October 15, 2020) showed a partial response of brain metastasis, pulmonary nodule, portal lymph node metastasis and peritoneal lymph node metastasis (Fig. 1囚-区). CEA, CA125, and CA153 all returned to normal level. The improvement of quality of life of the patient depended on the recovery of muscle tension. In November 2020, the patient developed melena without obvious cause, and gastroscopy showed compound ulcer, so he stopped taking trimetinib. The patient eventually died of gastrointestinal bleeding. He had progression-free survival (PFS) of 8 months of trimetinib monotherapy.

\section{Discussion And Conclusions}

Non-small-cell lung cancer (NSCLC) including squamous cell carcinoma, adenocarcinoma and large cell carcinoma accounts for $80-85 \%$ of lung cancer ${ }^{[1]}$. The incidence of brain metastasis (BMs) rate has started to climb sharply in recent years and $5-10 \%$ of NSCLC patients may develop BMs ${ }^{[2]}$. Early BMs are usually asymptomatic and the major symptoms of BMs including headache, vomiting, dizziness, diplopia, ataxia, hemiplegia and epileptic seizures ${ }^{[3]}$. Such patients have short survival period, poor quality of life and adverse prognosis. Surgical resection and radiotherapy are the main treatment options. In recent years, the strategies for the diagnosis and treatment of NSCLC have been gradually transformed into a precise target treatment era with the development of tumor molecular biology and genomics. There are a number of emerging therapeutic biomarkers and therapeutic concepts that show promise. BRAF mutation mostly was observed in lung adenocarcinoma, accounting for about $4.9 \%$. It mainly including V600E mutation and other rare mutations such as L956V and G468A, among which non-V600E positive accounted for about $43.3 \%{ }^{[4]}$. In June 2017, Dabrafenib in combination with trametinib were approved for the treatment of advanced NSCLC with BRAF V600E positive ${ }^{[5]}$. Gautschi $O$ et al ${ }^{[6]}$ believed that BRAF non-V600E positive were only found in smokers and the BRAF inhibitor darafenib was ineffective. No cases of BRAF non-V600E positive NSCLC with BMs have been reported. The genetic sequencing of this never-smoker patient is the BRAF exon D594N mutation, which is a rare mutation. We tried to treat with 
trimetinib monotherapy instead of combining with dalafenib or whole brain radiotherapy based on the patient's performance status, previous treatment and compliance. Trametinib is a MEK1/2 inhibitor which blocks MEK1/2 kinase activity and prevents RAF-dependent MEK phosphorylation ${ }^{[7]}$. It was approved initially as a monotherapy in treatment of advanced BRAF V600-mutant melanoma ${ }^{[8]}$. This patient was postoperative recurrence NSCLC with brain metastasis and multiple lymph nodes metastasis, who had been developed hemiplegia and nervous system symptoms. Multiple brain and lymph nodes lesions were gradually regressed during the treatment of trametinib. The patient had survived the 8 months of treatment and his quality of life was good. It is suggested that trimetinib can effectively control intracranial lesions by crossing the blood-brain barrier, and the anti-tumor effect on systemic and intracranial lesions deserve further study. Major adverse reactions of trimetinib included rash, diarrhea, hypertension, nausea, fatigue, pneumonia and fever, while rare adverse reactions included hemorrhage, venous thrombosis and cardiomyopathy ${ }^{[9]}$. In this case, grade 2 skin adverse reactions occurred in a short period of time, and no specific adverse events during the treatment. All toxicities were managed by positive measures. However, the patient later developed complex ulcerative gastrointestinal bleeding, leading to discontinuation of medication. It cannot be ruled out whether it was an adverse drug reaction considering the patient's previous history of gastric ulcer.

In conclusion, in the present non-smoking patient whose tumor showed a BRAF non-V600E positive, a partial response was observed despite brain and multiple lymph nodes lesions after trimetinib monotherapy. Trimetinib could potentially be a promising therapeutic agent for chemotherapy-refractory BRAF non-V600E mutant NSCLC patients. This case may help physicians develop a strategy for optimizing their approach to NSCLC. Nevertheless, in order to obtain more scientific experimental results, a large sample and high-quality randomized controlled trial is still needed to confirm the conclusions of this study.

\section{Abbreviations}

NSCLC: non-small-cell lung cancer; CT: computed tomography; MRI: Magnetic resonance imaging; PFS: progression-free survival; BMs: brain metastasis.

\section{Declarations}

Ethics approval and consent to participate: This study was approved by the Medical Ethics Committee of Jiangyin People's Hospital.

Consent for publication: Written informed consent was obtained from the patient and his son for publication of this case report and any accompanying images. A copy of the written consent is available for review by the Editor-in-Chief of this journal.

Funding: This study was not supported by any funding. 
Availability of data and materials: The datasets used and/or analysed during the current study are available from the corresponding author on reasonable request. T. P. and X. H. will make the data available to readers.

Competing interests: The authors declare that they have no competing interests.

Authors' contributions: X. Z. designed the study. T. P. collected data. T. P., X. Z. analyzed data and wrote the case report. T. P., X. Z. contributed to the discussion of results and to the review of the manuscript. All authors read and approved the final manuscript.

Acknowledgements: The authors would like to thank Dr. Xinjian Xu (Department of Interventional Radiology, Jiangyin People's Hospital, Jiangsu, China.) for advice and expertise.

Authors' information: ${ }^{1}$ 2Department of Interventional Radiology, Jiangyin People's Hospital, No. 3, Yingrui Road, Jiangyin City, Jiangsu Province, 214400, China.

\section{References}

1. Didkowska J, Wojciechowska U, Manczuk M, et al. Lung cancer epidemiology: contemporary and future challenges worldwide. Ann Transl Med. 2016;4(8):150-61.

2. Addeo R, Rosa CD, Faiola V, et al. Phase 2 trial of temozolomide using protracted low-dose and whole-brain radiotherapy for non-small cell lung cancer and breast cancer patients with brain metastases. Cancer. 2010;113(9):2524-31.

3. De DR, Dingemans AC, Praag J, et al. Prophylactic cranial irradiation versus observation in radically treated stage III non-small cell lung cancer: A randomized phase III NVALT-11/DLCRG - 02 study. J Clin Oncol. 2018;36(23):2366-77.

4. Marchetti A, Felicioni L, Malatesta S, et al. Clinical features and outcome of patients with non- small cell lung cancer harboring BRAF mutations. J Clin Oncol. 2011;29(26):3574-9.

5. Khunger $A$, Khunger $M$, Velcheti $V$. Dabrafenib in combination with trametinib in the treatment of patients with BRAF V600-positive advanced or metastatic non-small cell lung cancer: clinical evidence and experience. Ther Adv Respir Dis. 2018;12(1):1-9.

6. Gautschi O, Pauli C, Strobel K, et al. A patient with BRAF V600E lung adenocarcinoma responding to vemurafenib. J Thorac Oncol. 2012;7(10):23-4.

7. Kelly RJ. Dabrafenib and trametinib for the treatment of non-small cell lung cancer. Expert Rev Anticancer Ther. 2018;18(11):1063-8.

8. Flaherty KT, Robert C, Hersey P, et al. Improved survival with MEK inhibition in BRAF-mutated melanoma. N Engl J Med. 2012;367:107-14.

9. Blumenschein GR, Smit EF, Planchard D, et al. A randomized phase II study of the MEK1/MEK2 inhibitor trametinib (GSK1120212) compared with docetaxel in KRAS-mutant advanced non-smallcell lung cancer (NSCLC). Ann Oncol. 2015;26(5):894-901. 


\section{Figures}
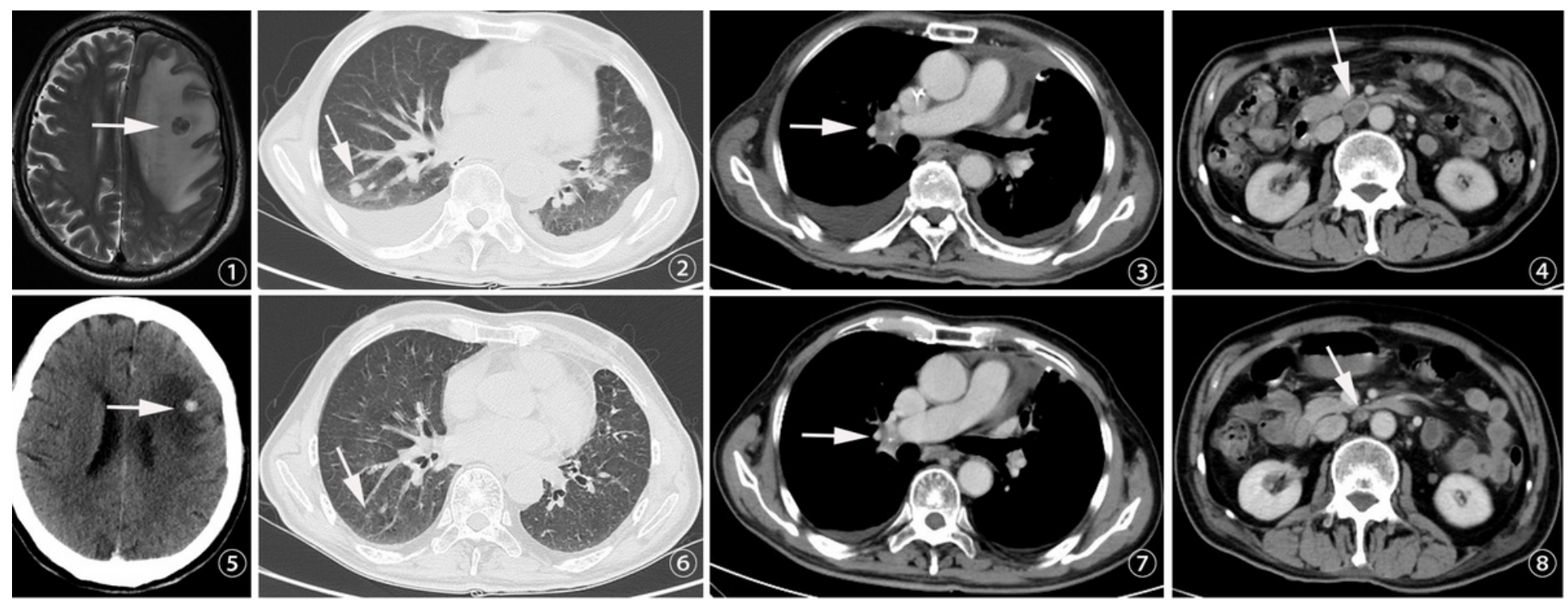

Figure 1

MR and CT imaging of a 71-year-old male with NSCLC before and after the therapy. $₫-\otimes$ The scan showed brain metastasis, pulmonary nodule, portal lymph node metastasis and peritoneal lymph node

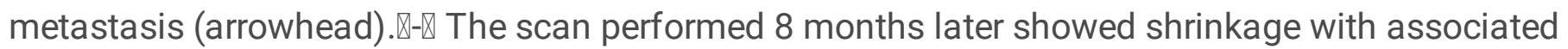
lesions (arrowhead).

\section{Supplementary Files}

This is a list of supplementary files associated with this preprint. Click to download.

- CAREchecklistEnglish2013.pdf 